\title{
Extensive skin color change caused by extravasation of indigo carmine
}

\author{
Ji-Won Choi ${ }^{1}$, Jeong Jin Lee ${ }^{1}$, Gunn-Hee Kim² ${ }^{2}$ and Seung-Hwan Hong ${ }^{2}$ \\ Department of Anesthesiology and Pain Medicine, ${ }^{1}$ Samsung Medical Center, ${ }^{2}$ National Medical Center, Seoul, Korea
}

Indigo carmine has been widely used in the clinic and its safety has been proven, but adverse reactions have been continuously reported [1-3]. We encountered a case of accidental extravasation of indigo carmine into a patient's forearm during general anesthesia.

A 72-year-old, $56 \mathrm{~kg}$, American Society of Anesthesiologists physical status II man was scheduled for robot-assisted laparoscopic radical prostatectomy for prostate cancer. His medical history was significant for chronic obstructive pulmonary disease and he had no known allergies. He arrived in the operating room with an 18-gauge peripheral intravenous (IV) line in his left forearm. Noninvasive blood pressure cuff, electrocardiography and pulse oximetry were applied. The pulse oximetry was applied on his right index finger. Anesthesia was induced with thiopental (275 mg) and rocuronium (50 mg), and endotracheal intubation was performed. Then, a 16-gauge peripheral IV line was placed in the right forearm and a radial arterial catheter was placed in the left radial artery. IV lines were working well and there were no swellings surrounding the IV insertion site. The patient position was lithotomy with Trendelenburg $\left(30^{\circ}\right)$ and both arms were placed beside the patient's body. Both arms were wrapped with a cotton roll to prevent hypothermia during surgery and were firmly attached to the bed using plaster.

Anesthesia was maintained with isoflurane and continuous infusion of remifentanil. Remifentanil was infused through the left IV line. Hartmann's solution was administered through the same line at a rate of $200 \mathrm{ml} /$ hour and normal saline was infused in the right IV line at a rate of $100 \mathrm{ml} /$ hour.

Two hours after induction, $5 \mathrm{ml}$ of $0.8 \%$ indigo carmine ( 40 $\mathrm{mg}$ ) was administered to the right side IV line according to the surgeon's request. However, 30 minutes after the injection of dye, the effect was not seen in the operating field, so the surgeon requested a repeated dose of indigo carmine injection. When we tried to inject the second time, we noticed that fluid administration via the right side IV line was slow. We tried to confirm the patency of the IV line by injecting a bolus of $10 \mathrm{ml}$ of normal saline but the speed of fluid administration did not increase. Suspecting a block in the IV line, no more fluid was administered. The patient's arm was covered and underneath the operation field, and therefore approach to it was difficult. There were no change in the wave of the pulse oximetry and total fluid administered through that IV was $200 \mathrm{ml}$ so we deduced that compartment syndrome did not occur. The indigo carmine was administered via the left side IV line and 5 minutes later the dye was confirmed at the surgical field.

The surgery ended approximately 3.5 hour after the first dye injection. During the operation the patient's blood pressure, heart rate and peripheral oxygen saturation were within normal range. Also, the arterial blood gas analysis implemented after the second dye injection were within normal range. The patient was awakened and trachea extubation was done. After the surgical drapes were removed, we noticed that the entire right forearm was blue and swollen. The range of discoloration was from the elbow to the dorsal part of the hand and the discoloration was distinct around the venous line (Fig. 1A).

Corresponding author: Jeong Jin Lee, M.D., Department of Anesthesiology and Pain Medicine, Samsung Medical Center, Sungkyunkwan University School of Medicine, 50, Ilwon-dong, Gangnam-gu, Seoul 135-710, Korea. Tel: 82-2-3410-2467, Fax: 82-2-3410-0362, E-mail: jjeong. lee@samsung.com

() This is an open-access article distributed under the terms of the Creative Commons Attribution Non-Commercial License (http:// creativecommons.org/licenses/by-nc/3.0/), which permits unrestricted non-commercial use, distribution, and reproduction in any medium, provided the original work is properly cited. 

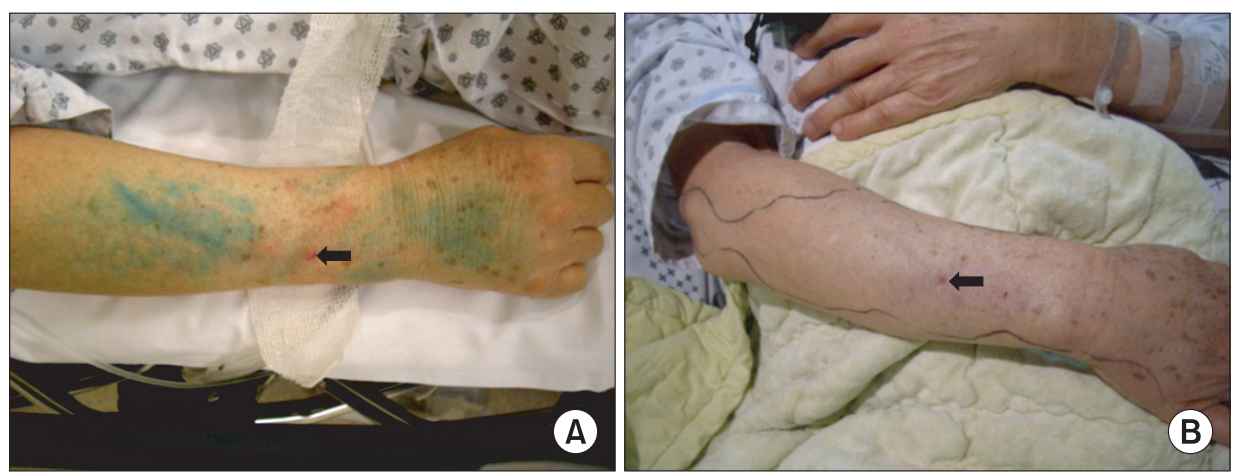

Fig. 1. Right forearm showing discoloration with indigo carmine. Arrow identifies the site of where the 16-gauge catheter was inserted (A). The black lines are the demarcation lines of the borders (B).

We did not have any prior information about the management of dye's extravasation, so we contacted the hospital pharmacy. While waiting for the answer, we transferred the patient to the postanesthesia care unit (PACU) and drew demarcation lines at the borders, applied an elastic bandage and elevated the affected arm. Motor and sensory function examinations were normal. The patient did not express any pain or discomfort. Cyanosis or dyspnea was not shown, so additional blood tests were not performed. Soon after, the hospital pharmacy informed that there had been a similar case before and that the discoloration resolved in 48 hours, but they did not know about tissue toxicity or delayed adverse reactions. In the PACU, we noticed that the discoloration started to disappear in a gravity-dependent fashion. We decided to observe the patient in the ward. Fifteen hours after transferring the patient to the ward, the discoloration disappeared and after 24 hours, it was completely reabsorbed (Fig. 1B).

O'Hara et al. [3] reported discoloration of the entire forearm after IV indigo carmine and total recovery in 48 hours in a patient who received vaginal hysterectomy. After arm elevation, the discoloration started to disappear from the elevated area, 24 hours later almost all the discoloration disappeared and after 48 hours, the discoloration was unnoticeable.

In our case, the discoloration disappeared within 24 hours without tissue toxicity or delayed adverse reaction. We can postulate that a larger amount of the dye had been extravasated than the report of O'Hara because they confirmed a change in the color of urine but we did not detect it. That means the dye failed to circulate the cardiovascular system and extravasated mostly to the subcutaneous area. Nevertheless, the reason why the discoloration in our case resolved in a short amount of time might be due to the relative height of the forearm and the heart. The patient's position was Trendelenburg $\left(30^{\circ}\right)$, so the patient's arm was located higher than the heart. Therefore, we assume that a large amount of the dye that had been extravasated had already been absorbed before we identified the discoloration of the patient.

Results of recent studies showed that conventional amounts of methylene blue dye used in chromoendoscopy and sentinel lymph node biopsy caused DNA damage but indigo carmine did not $[4,5]$. However, case reports of adverse reactions after IV or subareolar administration of indigo carmine have been reported. The adverse reactions were hypotension, hypoxemia and increased levels of methemoglobin $[1,2]$.

Thus, when there is an event of extravasation with indigo carmine subcutaneously, close observation precedes all others. After confirming that complications do not occur, reassurance to the patient that the discoloration will absorb naturally in 24 48 hours is recommended.

\section{References}

1. Hamman B. The adverse effects of indigo carmine. Anaesthesist 1989; 38: 136-9.

2. Yusim Y, Livingstone D, Sidi A. Blue dyes, blue people: the systemic effects of blue dyes when administered via different routes. J Clin Anesth 2007; 19: 315-21.

3. O'Hara JF Jr, Connor DF, Sprung J, Ballard LA. Upper extremity discoloration caused by subcutaneous indigo carmine injection. Anesth Analg 1996; 83: 1126-8.

4. Davies J, Burke D, Oliver JR, Hardie LJ, Wild CP, Routledge MN. Methylene blue but not indigo carmine causes DNA damage to colonocytes in vitro and in vivo at concentrations used in clinical chromoendoscopy. Gut 2007; 56: 155-6.

5. Masannat YA, Hanby A, Horgan K, Hardie LJ. DNA Damaging effects of the dyes used in sentinel node biopsy: possible implications for clinical practice. J Sur Res 2009; 154: 234-8. 